\title{
Applying Wavelet and Fourier Transform Analysis to Large Geophysical Datasets
}

\author{
Bjørn-Gustaf J. Brooks \\ Iowa State University, Ames IA 50011, USA \\ bjorn@climatemodeling.org
}

\begin{abstract}
The recurrence of periodic environmental states is important to many systems of study, and particularly to the life cycles of plants and animals. Periodicity in parameters that are important to life, such as precipitation, are important to understanding environmental impacts, and changes to their intensity and duration can have far reaching impacts. To keep pace with the rapid expansion of Earth science datasets, efficient data mining techniques are required. This paper presents an automated method for Discrete Fourier transform (DFT) and wavelet analysis capable of rapidly identifying changes in the intensity of seasonal, annual, or interannual events. Spectral analysis is used to diagnose model behavior, and locate land surface cells that show shifting cycle intensity, which could be used as an indicator of climate shift. The strengths and limitations of DFT and wavelet spectral analysis are also explored. Example routines in Octave/Matlab and IDL are provided.
\end{abstract}

\section{Introduction}

Many geophysical systems under study today are seasonal or operate on cycles in which particular states are revisited. Variation in systems that show recurrent states (e.g. climate change, the El Niño Southern Oscillation, Milankovitch cycles) is one of the most important topics in Earth science today. There exist a variety of mathematical methods for determining their dominant modes, among which Fourier and wavelet analysis both have the advantage of having common libraries and routines in several languages and software packages that can be used to develop automated routines to rapidly search for changes to the intensity and periodicity of cycles.

This paper uses an example dataset of temperature, precipitation, and soil moisture model output from the Parallel Climate Model (PCM). PCM is a fully coupled model. Ensembles of simulations were used to show global mean surface temperature increase of $\sim 1.9^{\circ} \mathrm{C}$ during the twenty-first century and a $3 \%$ increase in global precipitation[1]. A more complete description can be found in [2]. Figure 1 represents the 10 year running average of the 2796 land surface grid cells clustered into ecoregions by Hoffman and others[1], who used the descriptive variables of temperature, precipitation, and soil moisture to assign each land 


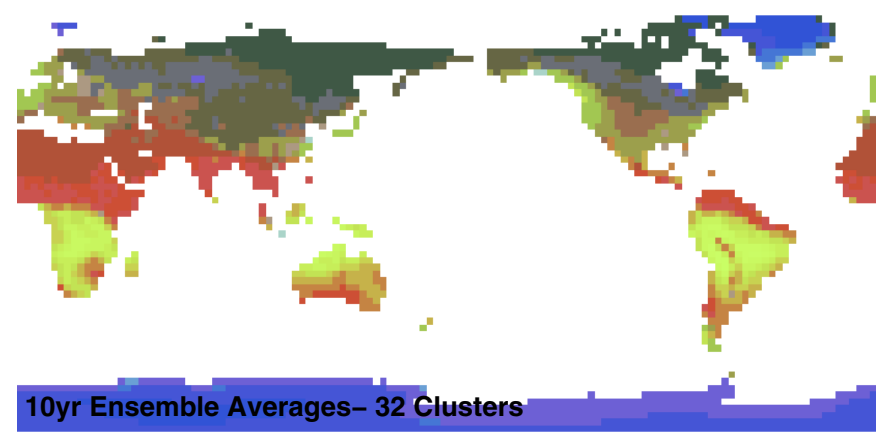

Fig. 1. Global map of 2796 PCM land surface cells taken from Hoffman and others [1]

surface cell to 1 of 32 ecoregion types through a 99 year (1188 month) Business As Usual (BAU) general circulation model (i.e. PCM). Three fields of ecological importance were analyzed: temperature $(\mathrm{K})$, precipitation $\left(\mathrm{kg} \mathrm{m}^{-2} \mathrm{~s}^{-1}\right)$, and soil moisture (fractional volume of root zone water ranging from 0:1).

The colors of each land surface cell in Figure 1 indicate the ecoregion type assigned by the Multivariate Spatio-Temporal Clustering (MSTC) procedure [1; 3], which was based on an iterative $k$-means algorithm[4]. Each land cell at each time point is described by three parameters of temperature, precipitation, and soil moisture that define its location in three-dimensional data space. Hoffman and others grouped these data points into clusters, called ecoregions, based on their euclidean distances. Climate changes for a land cell were said to have occurred when a land cell entered a climate regime that it had never previously visited.

To suit the purposes of this paper a scalable DFT routine was employed to identify particular PCM land surface cells that showed significant changes to the periodicity of their annual cycles. Two example land surface cells were selected for detailed wavelet analysis and to demonstrate how changes to the annual cycle within a time series of interest are located. The primary focus is to identify such variant land surface cells, which may serve as potential indicators of climate change.

\section{Discrete Fourier Transform Signal Detection}

Discrete Fourier transform analysis can be a useful technique for detecting periodic signals in time series [5]. Periodic signals within a time series are obtained by decomposing the time series into two parts, a real, $\Re\left\{F_{n}(x)\right\}$ and an imaginary, $\Im\left\{F_{n}(x)\right\}$. The power spectra of a DFT (Figure 2A) can be used to represent the cumulative magnitude of a signal within a time series. Changes to the spectral power of peaks within different window segments of a time series (e.g. a slackening annual cycle) can be identified by segmenting the time series into windows and computing the DFT power spectrum (DFTPS) for each window. When combined with Monte Carlo simulations, estimates for the significances of peaks in a power spectrum can also be determined. Example routines are available online at: www.climatemodeling.org/pcm/cyclicity/. 

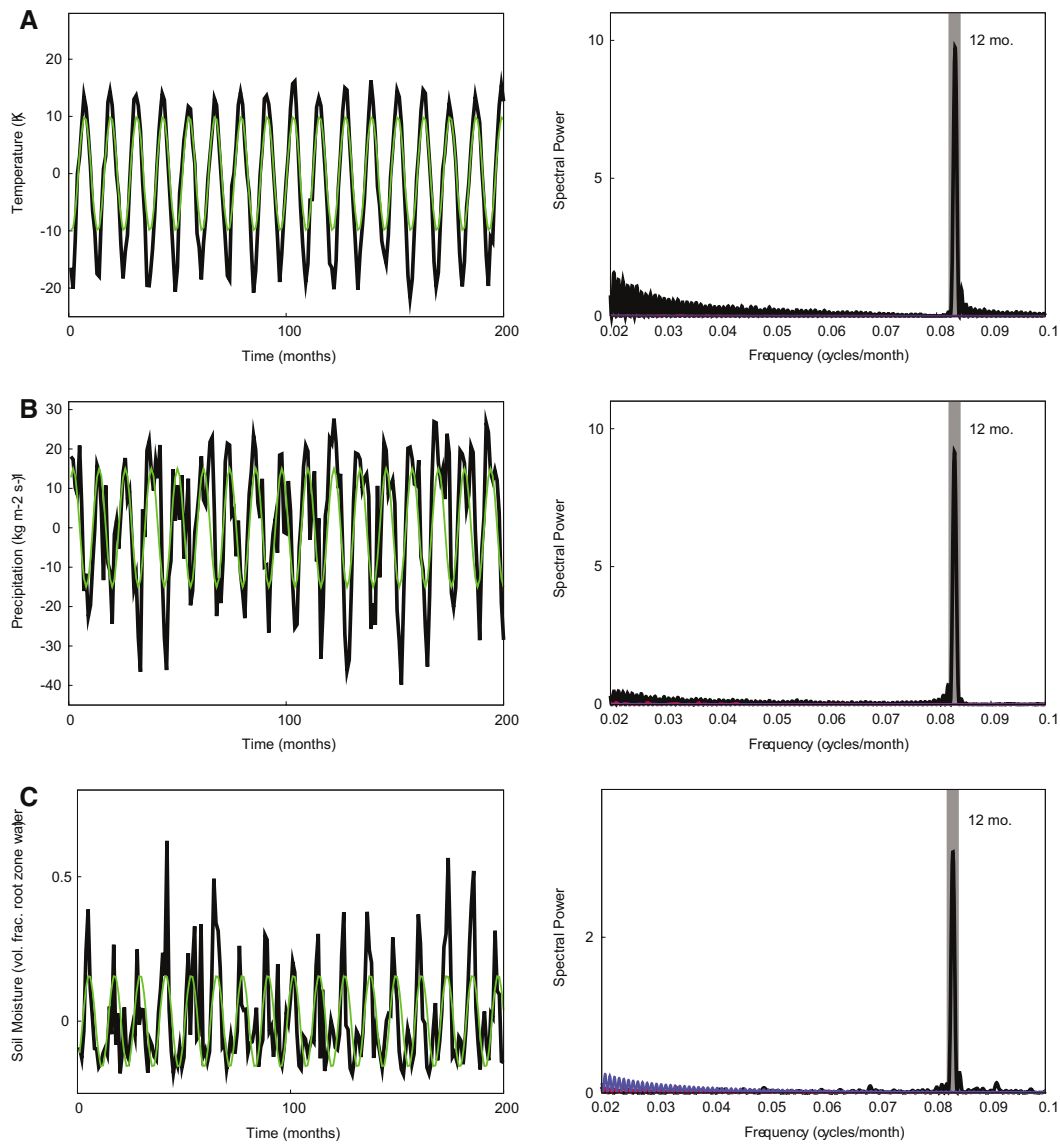

Fig. 2. Comparison of Discrete Fourier transform power spectrum (DFTPS) and time series plots. The DFTPS of three time series (mean subtracted) $\left\{\left(t_{i}, x_{i}\right)\right\}_{i=1}^{200}$ (soil moisture, precipitation, and temperature) of one Middle Eastern land cell from the Parallel Climate Model are shown in the left column with their corresponding DFT power spectra. Gray bars in the DFTPS locate the annual (12 month) period. Row A. represents temperature, B. precipitation, and C. soil moisture.

To compute the DFTPS in the second column of Figure 2, a 200 month segment of the original time series was extracted, $\left\{\left(t_{i}, x_{i}\right)\right\}_{i=1}^{200}$, which represents PCM precipitation data sampled at monthly increments $x_{i}$. The series was standardized to a variance of 1 , and then expressed as the sum of a series of sines and cosines through Fourier expansion. In its simplest form the DFT is denoted by Equation 1

$$
x(n)=\frac{1}{N} \sum_{k=0}^{N-1} X(k) e^{j 2 \pi n k / N}, n=0,1,2 \ldots N-1
$$




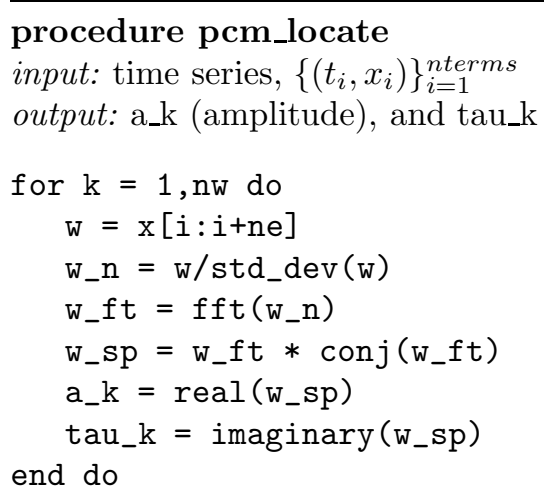

Fig. 3. The general procedure that locates windows within a time series with significant variation in the period and amplitude of the annual cycle. nw is the number of windows within the time series. ne is the number of elements $(\mathrm{x})$ within each window.

where $x(n)$ is the input time series at sample $n$, and $j=\sqrt{-1}$ is the basis for complex numbers. Many software packages have built in libraries and routines for DFT analysis and for producing DFTPS.

Figure 2 represents the monthly PCM output of temperature, precipitation, and soil moisture for one land surface cell. The left column represents the time series of the first 200 months of temperature, precipitation, and soil moisture for that same grid cell. The right column of DFT power spectra show the DFTPS of cycles within the three time series. The DFTPS each have one dominant cycle near $f=0.083$ (12 months), and can be used to answer two types of questions: 'What are the periods of cycles in the time series' and 'What are their significances?'

A well known limitation of Fourier expansion is that it has no time resolution meaning that although DFTPS indicate the frequencies present in a signal, nothing is indicated about where they are present in that signal or how their intensity changes through time. To avoid such problems a routine a routine was developed (pcm_locate.m) that divides each time series into window segments and computes their DFTPS to search for changes to the intensity and duration of the annual annual cycle (Figure 3). The results of all the land surface cells were sorted to identify particular cells that show dramatic change in their annual cycle. This routine can be scaled to run on a cluster.

\section{Wavelets}

Unlike Fourier expansion, wavelet functions broaden time series analysis into time-scale space, which allows wavelet methods to detect periodicities that are intermittent throughout the dataset [6]. Wavelet transform slides a window along 
the signal calculating the spectrum at each position, solving the time-domain limitation of Fourier expansion by a method more efficient that windowing $[7]$. Wavelet spectrograms can be used as representations of time and frequencydomain changes for the variant land surface cells once identified.

Wavelet functions $(\psi)$ concern the relationship between a dilation parameter $S$, also called scale, and a translation parameter $\tau$, also called shift and are described by Equation 2 .

$$
\Psi_{S, \tau}(\eta)=\frac{1}{\sqrt{S}} \Psi\left(\frac{\eta-\tau}{S}\right)
$$

$\eta$ is a nondimensional sampling parameter, and $S^{-1 / 2}$ normalizes the energy across different scales, which allows the spectral power of a signal to be compared across different scales. Equation 2 shows a key distinction of wavelet analysis in that it does not specify a wavelet basis function. This flexibility permits different wavelet functions to be used. Changing the wavelet function or varying the parameters that accompany the wavelet function, for example the scale, has important effects on the resulting spectrogram. Although many studies use the Morlet wavelet function for its frequency resolution, in this paper the Paul function is used to provide better time resolution. A comparison of the uses of different wavelet functions can be found in [8].

Wavelet basis functions or wavelets have zero means (Figure 4) and are localized in time and Fourier space. Given an array of equally spaced observations, $x_{n}$, from $n=0 \ldots N-1$, a continuous wavelet function, $\psi(\eta)$, can detect variations in the power of an array of data $[9]$.

As in Fourier analysis the resulting wavelet power spectrum is partitioned into a real part (the amplitude) and an imaginary part (the phase information). The key distinction of wavelet transform is that the period and spectral power of the wavelet transform can be plotted through time (Figure 5). Strongly cyclic events are indicated by hot colors on the spectrogram, while small peaks and
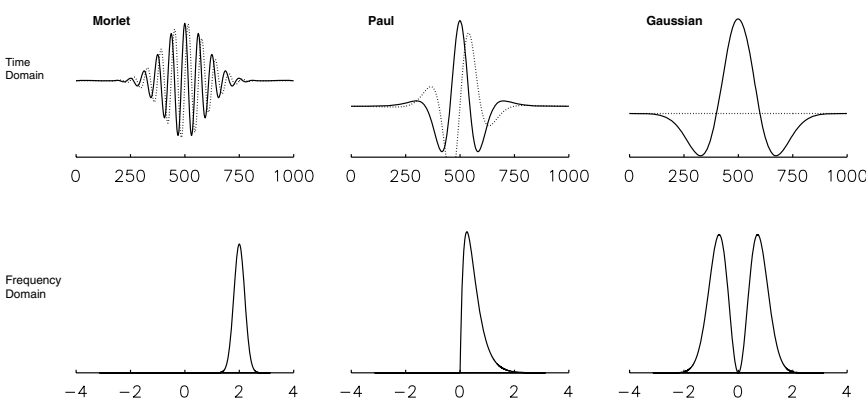

Fig. 4. Wavelet functions. The plots in the upper row indicate the real (solid) and imaginary (dotted) parts in the time domain, while the bottom row corresponds to the frequency domain of those same wavelet functions. 

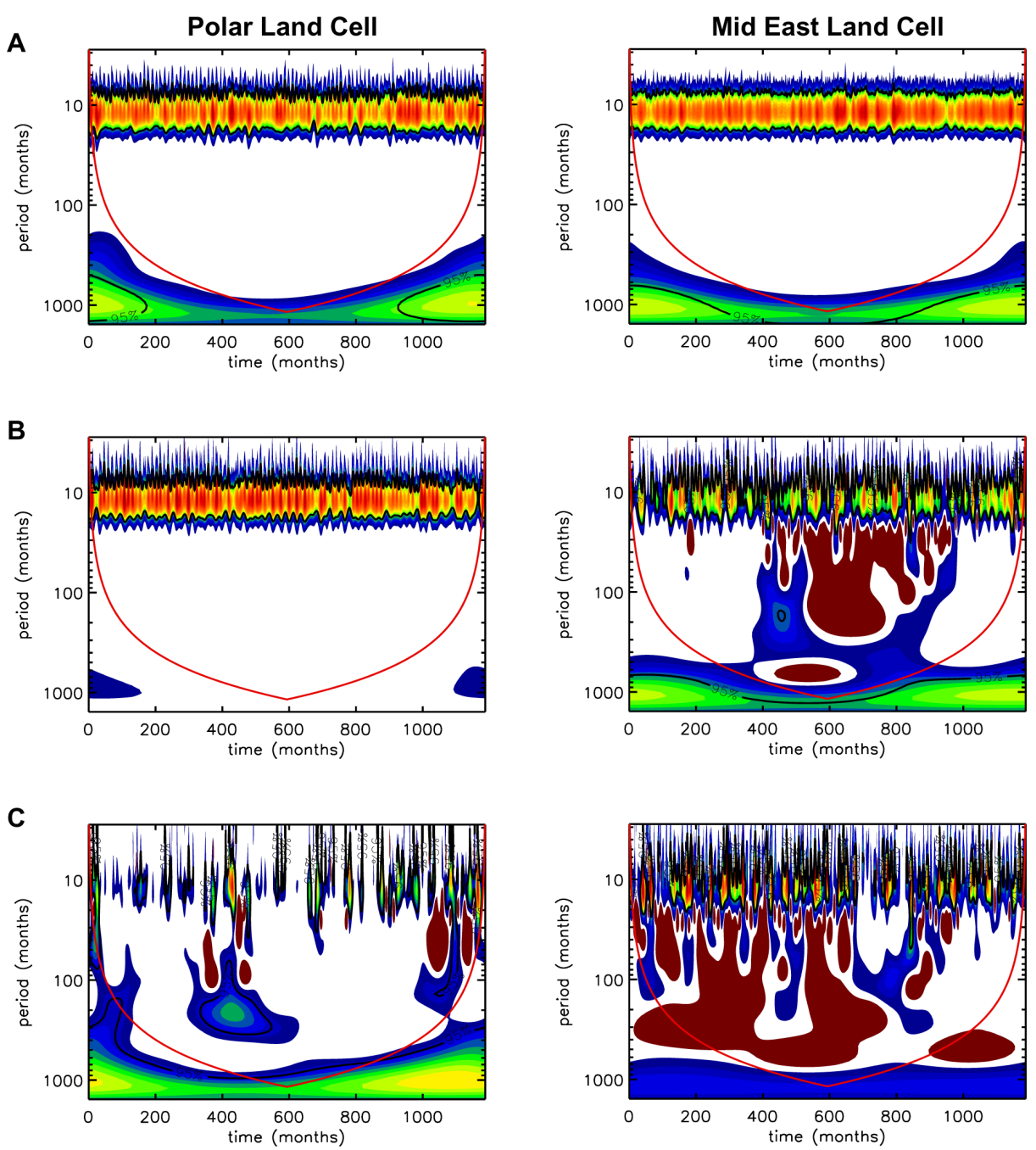

Fig. 5. Paul wavelet spectrograms of two land surface cells, divided into left (a typical polar cell) and right (a particularly variant Mid East cell) columns. Hot colors (red) indicate high spectral power (a strong cycle), whereas blue indicates little spectral intensity (a weak cycle). A. represents the temperature data for both cells. B. the precipitation, and C. soil moisture. The cone of influence is shown by the red curve, and a $95 \%$ confidence interval is indicated for the annual cycle by the black line encircling it.

weak cycles are indicated by cool colors. These plots can be reproduced using

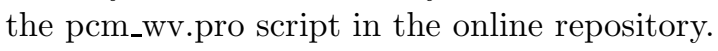

Because a continuous wavelet transform of a finite length time series has edge artifacts near the beginning and end of the power spectrum that represent an incomplete localization of the data, a cone of influence (COI) is used in wavelet 
spectrograms to represent the threshold below which the significance of cycles is dubious [6]. However, if dealing with geographic data, such as for a band of longitude with fixed latitude, the data are continuous (i.e. they wrap around) and no COI is necessary.

\section{Monte Carlo Statistical Significances}

Although a spectral peak may appear in the data, it could be a computational artifact. Monte Carlo (MC) simulations can be used as null models to determine the statistical significances of peaks within a power spectrum. For any time series there are frequencies within which peaks are likely to be the result of random chance. MC trials are one way to decipher the significance of spectral peaks from random chance. Two MC routines are used as null models and the significance of spectral peaks in the original signal are computed by counting the number of times the amplitude of a peak generated by a random permutation of the data is able to exceed that of the original time series.

As opposed to purely theoretical (white noise) simulations, the two MC methods used here simulate the probability of occurrence of spectral peaks based on assumptions about the cause of the periodicity. These MC methods are adapted from Cornette[10], and Rohde and Muller[11]. They assume that fluctuations in the DFTPS follow an exponential form, such that the probability of spectral power at frequency $f$ would be $P(h)=e^{-h / h(f)}$ where $h(f)$ denotes the average height $h$ of the power spectrum at frequency $f$ across many trial simulations.

In the first method, random walk trials, at each time increment $t_{i}$, a data point $x_{i}$ is randomly selected from the series and used to generate a permutation of the original sequence $\left\{\left(t_{i}, x_{i}\right)\right\}_{i=1}^{1188}$. This represents the null hypothesis that diversity is a random walk. It is important to reject that variations in the time series are not best described by a random walk for cases where the fundamental dynamics of a system are complex or poorly constrained. The plot of the mean of 10,000 random walk trials is shown by the red curve in the right column of Figure 2 .

For random block trials, the time series data are partitioned into $N$ blocks of length $M$. At each increment of $N$ the blocks are randomly permuted to obtain a random sequence. This method preserves some short term relationships between data points in the time series, and represents the hypothesis that variation in the time series is independently driven (i.e. directed) with major random perturbations. For the temperature, precipitation, and soil moisture PCM output a block length, $M$, of 12 (months) is used to represent the null model that seasonal trends are directed, but interannual variability is random. This is represented by the blue curve that appears in the right column of Figure 2 An example script for producing this plot can be found in our online repository (dftps.m).

Significance is computed for a peak of height $h$ at a frequency $f$, as the fraction, $p$, of the randomly generated sequences for which the MC spectrum at $f$ exceeds $h$. Table 1 lists the significance of the annual cycle in precipitation for the Middle Eastern cell in three different windows of the time series to show its evolution ( $c f$. Figure $5 \mathrm{~B}$ ). The probability that a MC simulation of the 12 month 
Table 1. The significances of the annual peak in the data from Figure $5 \mathrm{~B}$ (precipitation data for the Mid East cell) fluctuates throughout the time series. Statistical significances of the 12 month cycle relative to 10,000 random walk (RW) and random block (RB) Monte Carlo simulations are given below in four conterminous 48 month windows of the time series between 600 and 743 months.

\begin{tabular}{lccc}
$\begin{array}{c}\text { window } \\
\text { segment }\end{array}$ & $\begin{array}{c}\text { location in } \\
\text { time series }\end{array}$ & $\begin{array}{c}\text { RW } \\
\text { significance }\end{array}$ & $\begin{array}{c}\text { RB } \\
\text { significance }\end{array}$ \\
\hline \multicolumn{4}{c}{ precipitation } \\
One & $600-647$ & 0.002 & 0.001 \\
Two & $648-695$ & 0.003 & 0.000 \\
Three & $696-743$ & 0.043 & 0.032
\end{tabular}

cycle has a greater significance is very small $(p<0.005)$ in windows one and two. Segment three however, has a reduced significance that seems to indicate a slackened annual cycle near 700 months.

\section{Summary of Results}

Reliable climate change determinations require powerful analytical tools, among which spectral analysis can be included. Figure 1 shows an example output from Hoffman and others [1] MSTC technique, which assigned each of the 2796 land cells to one of 32 climate regimes at each time point (month). Their evolution was tracked through time. Hoffman and others chose one representative Middle Eastern cell as an example that underwent a climate change by transitioning into the hottest and driest climate state, a regime it had never previously visited. The rigorousness of this climate transition can be explored by showing a shift in the intensity and duration of its annual cycles, for example in the duration of wet season precipitation events or in the intensity of peak summer temperatures.

While, temperature spectrograms for the polar and Middle East land cells are nearly identical (Figure 5A), substantial differences exist in soil moisture and precipitation. The first 600 months of soil moisture data for the Middle East land cell contrasts the last 600 months in Figure $5 \mathrm{C}$. The first half is characterized by a strong annual soil moisture cycle, which are accompanied by a range of possibly significant 12-120 month interannual soil moisture cycles. The second half of the time series shows noticeably fewer of these interannual cycles and more frequent gaps in the annual soil moisture cycle. The low frequency (red and blue) cycles that appear below the annual period should be regarded with caution, as they have a limited recurrence and do not have statistical significances equal to that of the 12 month cycle.

A diminishing annual soil moisture cycle could be the result of two phenomena. It may be the consequence of changes in air temperature or precipitation. Little change appears throughout the temperature spectrogram, therefore is appears to be the latter case. 
Interestingly, the slackening of the precipitation cycle shown Figure $5 \mathrm{~B}$ roughly coincides with a shift in the annual precipitation cycle. However, changes to the soil moisture cycle appear more clear than those to the precipitation cycle, and this information could be related to the timing of the observed climate transition as determined by clustering, and it could be used to understand whether the shift was one primarily of one or two environmental parameters. This would be especially useful for examining land surface cells that have strong seasonal precipitation events.

Spectral analysis techniques can be a useful means to rapidly identify variant periodicities in geophysical time series, and to diagnose the behavior of model simulations. The periodicity captured by model output, and especially the change in duration and intensity of cycles, is an important feature that can be rapidly analyzed using the methods outlined in this paper.

\section{Bibliography}

[1] Hoffman, F.M., Hargrove, W.W., Erickson, D.J.: Using clustered climate regimes to analyze and compare predictions from fully coupled General Circulation Models. Earth Interactions 9(10), 1 (2005)

[2] Washington, W.M., Weatherly, J.W., Meehl, G.A., Semtner Jr., A.J., Bettge, T.W., Craig, A.P., Strand Jr., W.G., Arblaster, J., Wayland, V.B., James, R., Zhang, Y.: Parallel climate model (PCM) control and transient simulations. Climate Dynamics 16, 755-774 (2000)

[3] Hoffman, F.M., Hargrove, W.W.: Multivariate geographic clustering using a Beowulf-style parallel computer. In: Arabnia, H.R. (ed.) Proc. International Conference on Parallel and Distributed Processing Techniques and Applications (PDPTA 1999), Las Vegas, Nevada, vol. III, pp. 1292-1298. CSREA Press (June 1999)

[4] Hartigan, J.A.: Clustering Algorithms. John Wiley \& Sons, New York (1975)

[5] Cadzow, J.A.: Discrete-Time Systems: An Introduction with Interdisciplinary Applications. Prentice-Hall, Inc., Englewood Cliffs (1973)

[6] Torrence, C., Compo, G.P.: A practical guide to wavelet analysis. Bulletin of the American Meteorological Society 79, 61-78 (1998)

[7] Kaiser, G.: A Friendly Guide to Wavelets. Birkhäuser, Boston (1994)

[8] De Moortel, I., Munday, S.A., Hood, A.W.: Wavelet analysis: the effect of varying basic wavelet parameters. Solar Physics 222, 203-228 (2004)

[9] Farge, M.: Wavelet transforms and their applications to turbulence. Annual Review of Fluid Mechanics 24, 395-457 (1992)

[10] Cornette, J.L.: Gauss-vaníček and fourier transform spectral analyses of marine diversity. Computing in Science and Engineering 9(4), 61-63 (2007)

[11] Rohde, R.A., Muller, R.A.: Cycles in fossil diversity. Nature 434, 208-210 (2005) 